\title{
Caracterización mediante HRTEM de un acero AISI 4140 nitrurado por postdescarga micro-ondas
}

\author{
L. Béjar-Gómez", A. Medina-Flores**, M. Pérez-López* y C. Maldonado-Zepeda**
}

Resumen Un análisis de las fases de nitruros formados en un acero AISI 4140, nitrurado mediante condiciones de postdescarga micro-ondas, se llevó a cabo por microscopia electrónica de transmisión de alta resolución (HRTEM), rápida transformada de Fourier (FFT) y nanoanálisis. Las muestras de acero fueron nitruradas por debajo del punto de transformación eutectoide $\left(590^{\circ} \mathrm{C}\right)$; después del tratamiento de nitruración las piezas fueron enfriadas lentamente dentro del reactor con una atmósfera de argón. Los análisis se realizaron en un microscopio Philips con cañón de emisión de campo Tecnai F20 a $200 \mathrm{Kv}$ (PHILIPS FEG-TEM TECNAI F20). Los resultados mostraron la morfología y tamaño de los nitruros de hierro $\varepsilon-\mathrm{Fe}_{2-3} \mathrm{~N}$ y $\gamma^{\prime}-\mathrm{Fe}_{4} \mathrm{~N}$, los cuáles mostraron coherencia con la matriz. Además, se obtuvo la siguiente relación de coherencia entre precipitados y matriz: (110) $\mathrm{Fe}-\alpha\left\|(1001) \varepsilon-\mathrm{Fe}_{2-3} \mathrm{~N}\right\|(110) \gamma^{\prime}-\mathrm{Fe}_{4} \mathrm{~N}$ y $\left[\begin{array}{lll}1 & -1 & 0\end{array}\right] \mathrm{Fe}-\alpha\left\|\left[\begin{array}{llll}1 & 0 & -1 & 0\end{array}\right] \varepsilon-\mathrm{Fe}_{2-3} \mathrm{~N}\right\|\left[\begin{array}{llll}0 & 1 & 1\end{array}\right] \mathrm{Fe}_{4} \mathrm{~N}$.

Palabras clave HRTEM. Nitruros de hierro. Nanoanálisis. FEG. FFT.

\section{Characterization for HRTEM of AISI 4140 steel nitrided by postdischarge micro-wave}

\begin{abstract}
An analysis of the nitrides formed in an AISI 4140 steel nitrided by postdischarge microwave nitriding treatment was carried out by high resolution transmission electron microscopy (HRTEM), fast Fourier transform (FFT) and nanoanalysis. The steel samples were nitrided below of the eutectoid transformation point $\left(590^{\circ} \mathrm{C}\right)$, after nitriding the samples were cooling slowly inside in the reactor whit an argon atmosphere. The analysis were carried out in a FEG-TEM PHILIPS TECNAI F20 at $200 \mathrm{Kv}$. The results showed the morphology and size of the nitrides $\varepsilon-\mathrm{Fe}_{2-3} \mathrm{~N}$ y $\gamma^{\prime}-\mathrm{Fe}_{4} \mathrm{~N}$, which had coherency whit the matrix. By other hand the following crystallographic relations were determined:
\end{abstract}

(110) Fe- $\alpha\left\|(1001) \varepsilon-\mathrm{Fe}_{2.3} \mathrm{~N}\right\|(110) \gamma^{\prime}-\mathrm{Fe}_{4} \mathrm{~N}$ y [ $\left[\begin{array}{lll}1 & -1 & 0\end{array}\right] \mathrm{Fe}-\alpha\left\|\left[\begin{array}{llll}1 & 0 & -1 & 0\end{array}\right] \varepsilon-\mathrm{Fe}_{2-3} \mathrm{~N}\right\|\left[\begin{array}{llll}0 & 1 & 1\end{array}\right] \mathrm{Fe}_{4} \mathrm{~N}$.

Keywords HRTEM. Iron nitrides. Nano-analysis. FEG. FFT.

\section{INTRODUCCIÓN}

La nitruración es un tratamiento termoquímico utilizado en aceros para modificar las propiedades en la superficie por la difusión de nitrógeno. Dependiendo del proceso de nitruración y la composición de la acero, la zona de difusión del nitrógeno puede penetrar por encima de $0,7 \mathrm{~mm}$ dentro del acero, la capa compuesta formada sobre la superficie de la zona de difusión tiene espesores en el rango micrométrico. En la zona de difusión, la microestructura es cambiada por la introducción de átomos de nitrógeno intersticiales en solución sóli- da y cuando el límite de solubilidad se alcanza, precipitados muy finos y coherentes se forman, que dependiendo de los nitruros formados con los elementos de aleación, pueden hacer una contribución significativa a la fatiga de los componentes (tensión, compresión, torsión, doblez) ${ }^{[1]}$.

La nitruración por post-descarga micro-ondas es un método que ofrece ventajas sobre los métodos tradicionales de nitruración, ya que produce altas concentraciones de $\mathrm{N}^{+}$, que permite un rápido crecimiento cinético de nitruros ${ }^{[2]}$.

La microscopia electrónica de transmisión de alta resolución (HRTEM), se ha convertido en los

(*) Facultad de Ingeniería Mecánica. Edificio “W". Cd. Universitaria. Universidad Michoacana de San Nicolás de Hidalgo (UMSNH).

$\left.{ }^{* *}\right)$ Instituto de Investigaciones Metalúrgicas (IIM). Edificio "U". Cd. Universitaria. Universidad Michoacana de San Nicolás de Hidalgo (UMSNH). C.P. 58000 Tel. (+52)-443-3040747, Fax. (+52)-443-3162934, Morelia, Michoacán. México. E-mail: ibgomez@zeus.umich.mx ariosto@jupiter.umich.mx, maxperez@yahoo.com.mx 
últimos años en la herramienta más importante para el análisis estructural de nanocristales por la resolución que brinda, con lo que es posible observar una imagen a manera de proyección de las columnas de átomos alineadas en el mismo sentido del haz de electrones incidente ${ }^{[3 \text { y } 4]}$, lo que permite obtener información detallada a nivel atómico, ya que presenta información de las interfases, morfología de los diferentes precipitados, relaciones cristalográficas entre los precipitados y la matriz ${ }^{[5]}$.

La digitalización de imágenes es una herramienta importante en el estudio de estructuras cristalinas, y se realiza mediante el procesamiento digital de imágenes, que permite procesarlas en espacio real y espacio recíproco, filtrando las correspondientes frecuencias de cada fase en la muestra. Con este proceso la estructura de la muestra observada puede realzarse ${ }^{[6]}$.

En este trabajo se aborda el análisis para la determinación de las estructuras y composición de arreglos locales de granos cristalinos de los nitruros de hierro formados después del tratamiento de nitruración por post-descarga micro-ondas llevado a cabo mediante microscopia electrónica de transmisión de alta resolución (HRTEM), transformada rápida de Fourier (FFT) y nanoanálisis realizados mediante la técnica de EDS.

\section{MATERIALES Y PROCEDIMIENTO EXPERIMEN- TAL}

\subsection{Material}

Se utilizó un acero comercial AISI 4140, el cual tiene la composición química mostrada en la tabla I. El acero fue maquinado en cubos de $2 \times 2 \times 1 \mathrm{~cm}$. Después del maquinado las piezas fueron sometidas a un tratamiento de normalizado a $840^{\circ} \mathrm{C}$ durante 30 min y enfriadas lentamente al aire. Antes de aplicarles el tratamiento de nitruración, ambas caras fueron desbastadas en papel abrasivo, en lijas de $\mathrm{SiC}$ de diferente granulometría. Posteriormente, fueron pulidas a espejo, usando alúmina de 0,3 y $0,05 \mu \mathrm{m}$, y limpiadas en una tina de ultrasonido con acetona, para eliminar impurezas.

Tabla I. Composición química del acero AISI 4140

Table I. Chemical composition of AISI 4140 steel

\begin{tabular}{lcccccccc}
\hline Elemento & $\mathbf{C}$ & $\mathrm{Mn}$ & $\mathrm{Si}$ & $\mathbf{P}$ & $\mathbf{S}$ & $\mathrm{Cr}$ & $\mathrm{Mo}$ & $\mathrm{Ni}$ \\
\hline $\begin{array}{l}\text { Composición } \\
\text { (\% en peso) }\end{array}$ & 0,412 & 0,665 & 0,23 & 0,015 & 0,014 & 1,15 & 0,15 & 0,50 \\
\hline
\end{tabular}

\subsection{Caracterización mediante Microscopía Electrónica de Transmisión de Alta Resolu- ción (HRTEM)}

Antes de realizar la caracterización mediante microscopía electrónica de transmisión, las muestras fueron sometidas a un proceso de preparación con el objetivo de obtener zonas transparentes al paso de los electrones, consistente en: Se cortaron secciones transversales de las muestras nitruradas mediante una cortadora de disco de diamante; posteriormente, se desbastaron manualmente en lijas de diferente granulometría hasta obtener espesores de $70 \mu \mathrm{m}$; se unieron dos muestras desbastadas en la sección de la capa nitrurada mediante un pegamento de la marca Gatan; las secciones unidas se dejaron secar durante $36 \mathrm{~h}$ y, con la ayuda de un disco ultrasónico de corte, se obtuvieron discos de $3 \mathrm{~mm}$ de diámetro, como se observa en la figura 1 , que fueron desbastados mecánicamente hasta obtener espesores de $5 \mu \mathrm{m}$; finalmente, las muestras fueron desbastadas con iones de argón hasta obtener zonas transparentes al paso de los electrones. Para la caracterización de las muestras se utilizó un equipo FEG Tecnai F-20, con aberración esférica, $\mathrm{C}_{\mathrm{s}}=1 \mathrm{~mm}$, con un límite de información de $0,15 \mathrm{~nm}$, resolución máxima de 0,23 nm. Las imágenes de HRTEM se obtuvieron en forma digital, utilizando una cámara CCD (charge cuopled device) de barrido lento marca Gatan, que está acoplada al microscopio. Al digitalizarse las imágenes, quedaron codificadas como una matriz de intensidades $\mathrm{n} \times \mathrm{m}$. En forma digital se obtuvo la transformada

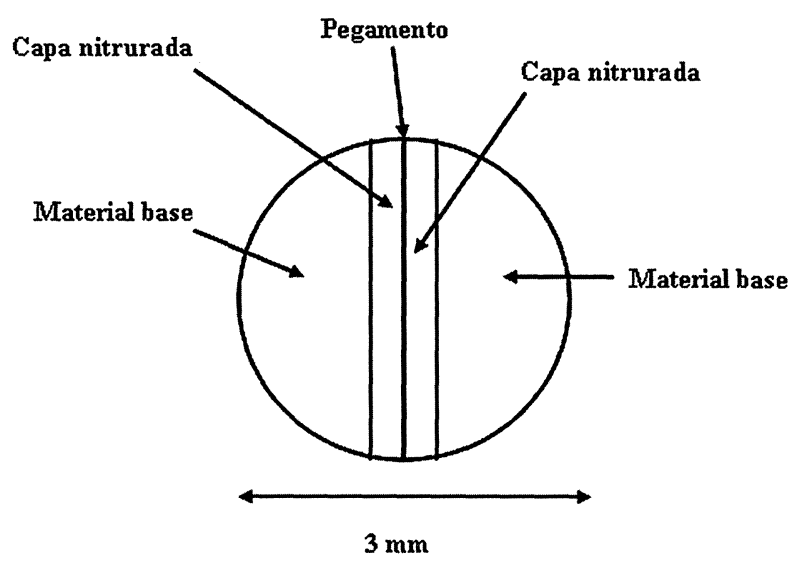

Figura 1. Imagen de la muestra obtenida mediante el disco ultrasónico de corte, mostrando las zonas unidas con el pegamento de gatan.

Figure 1. Sample image obtained by the ultrasonic disc cuter, showing the zones joined whit the gatan glue. 
Caracterización mediante HRTEM de un acero AISI 4140 nitrurado por postdescarga micro-ondas L. BÉJAR-Gómez, A. MEDINA-FloRes, M. PÉREz-LóPez y C. MALDONADO-ZEPEDA

rápida de Fourier (FFT) de las imágenes digitalizadas, que es proporcional a un patrón de difracción con el que fue posible determinar las frecuencias características de los componentes en la matriz cristalina y determinar la estructura de los cristales formados. Posteriormente, a la FFT se le aplicaron diferentes "máscaras" para filtrar las frecuencias espaciales y el ruido, y de esta manera, realzar su estructura cristalina correspondiente ${ }^{[3]}$. Finalmente, se adquirió la transformada rápida de Fourier inversa (IFFT), para obtener la imagen procesada final. Los nanoanálisis fueron obtenidos con un tiempo de adquisición de $100 \mathrm{~s} \mathrm{y} \mathrm{con} \mathrm{un} \mathrm{diámetro}$ del haz de 0,5 nm El equipo con el cual se realizó el análisis se encuentra ubicado en el Instituto de Investigaciones Metalúrgicas (IIM) perteneciente a la Universidad Michoacana de San Nicolás de Hidalgo (UMSNH).

\section{RESULTADOS Y ANÁLISIS DE RESULTADOS}

\subsection{Caracterización mediante Microscopía Electrónica de Transmisión (HRTEM) del los nitruros $\gamma^{\prime}-\mathrm{Fe}_{4} \mathrm{~N}$ y $\varepsilon-\mathrm{Fe}_{2-3} \mathrm{~N}$}

Los resultados que se habían obtenido anteriormente mediante diferentes técnicas de caracterización mostraron, solamente, la formación de 2 fases diferentes de nitruros de hierro en la superficie del material: $\gamma^{\prime}-\mathrm{Fe}_{4} \mathrm{~N}$ y $\varepsilon-\mathrm{Fe}_{2-3} \mathrm{~N}$. Por lo tanto, resultaba procedente obtener su morfología, estructura cristalina y composición química a nivel nanoestructural. La caracterización de la fase cristalina se obtiene de la imagen directa y su correspondiente FFT, el cual muestra el espaciamiento de red.

\subsection{Caracterización del nitruro $\gamma^{\prime}-\mathrm{Fe}_{4} \mathrm{~N}$}

La figura 2 muestra una imagen del nitruro $\gamma^{\prime}-\mathrm{Fe}_{4} \mathrm{~N}$ después de aplicarle la IFFT obtenida sobre la capa nitrurada, mostrando las diferentes distancias interatómicas de los diferentes planos atómicos paralelos, mostrando un distanciamiento interplanar de $0,268 \mathrm{~nm}$ que coincide con las tablas JCPDSICDD para este nitruro.

La imagen de la FFT mostrada en esta misma figura, en el lado superior derecho, muestra un arreglo puntual cuadrado, con sus principales planos marcados y sus correspondientes valores. $\mathrm{Al}$ indexar la FFT se determinó que la estructura cristalina de nitruro es cúbica centrada en las caras, obteniéndose un eje de zona cercano a la orientación $[0,0, \overline{1}]$.

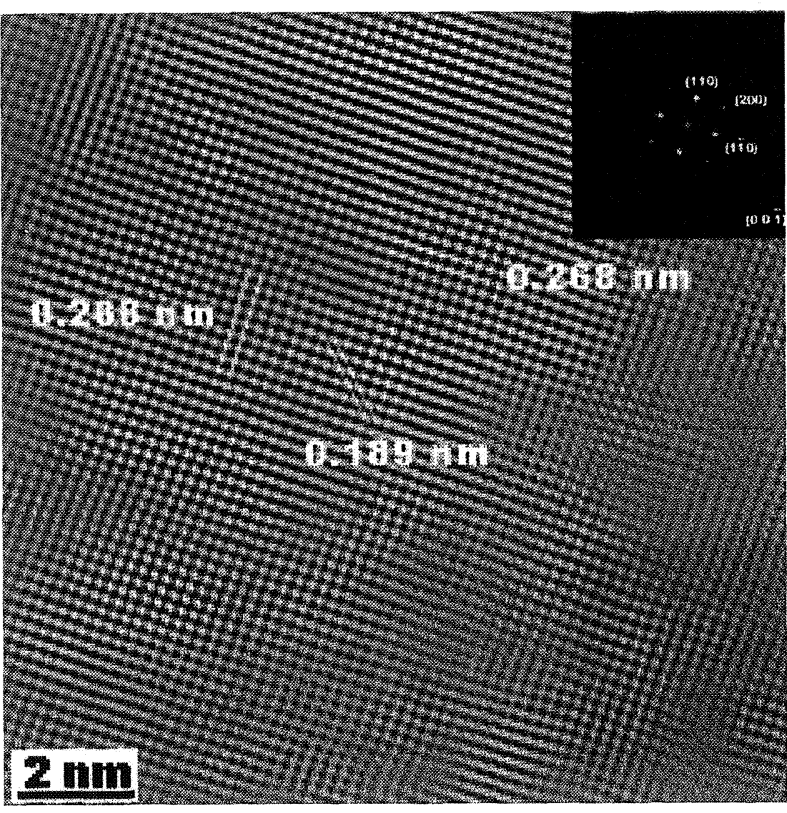

Figura 2. Imagen de la IFFT obtenida por HRTEM del nitruro $\gamma^{\prime}-\mathrm{Fe}_{4} \mathrm{~N}$, mostrando los valores de las distancias interatómicas en diferentes direcciones de planos paralelos de 0,268 $\mathrm{nm}$ que corresponden a la distancia entre los planos (110) y Transformada Rápida de Fourier (FFT) en el eje de zona $[0,0,1]$.

Figure 2. IFFT HRTEM image of the $\gamma-\mathrm{Fe}_{4} \mathrm{~N}$ nitride showing the interatomic distances in several directions of parallel planes of $0.268 \mathrm{~nm}$ that correspond to the distance between (110) planes and Fast Fourier Transform (FFT) in the $[0,0,1]$ axis zone.

La figura 3 muestra el espectro de energía dispersiva (EDS) del nanoanálisis de la figura 2, en la cual se observa la presencia de los picos de nitrógeno y hierro. La tabla II muestra la cuantificación del espectro. Es importante mencionar que el valor del nitrógeno es de 19,434 \% atómico, el cual esta de acuerdo con lo expuesto por R.C. Rhul y $\mathrm{M}$ Cohen, en su tabla resumida, para las diferentes fases que constituyen el sistema Fe-N.

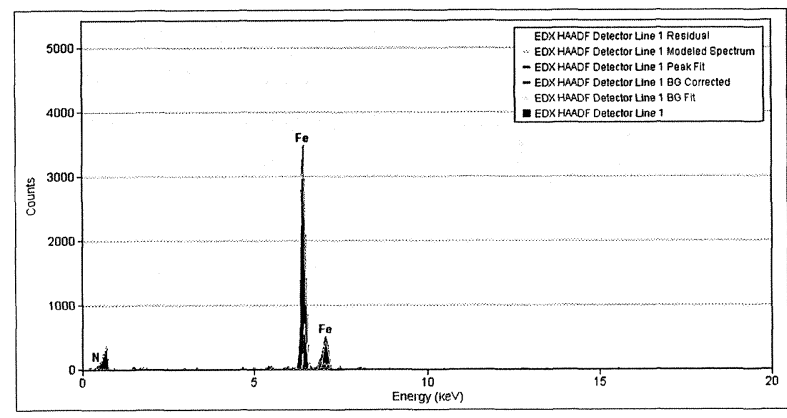

Figura 3. Espectro de nanoanálisis de la fase $\gamma-\mathrm{Fe}_{4} \mathrm{~N}$.

Figure 3. Nanoanalysis spectrum of the $\gamma-\mathrm{Fe}_{4} \mathrm{~N}$ phase. 
Caracterización mediante HRTEM de un acero AISI 4140 nitrurado por postdescarga micro-ondas L. BÉJAR-GÓMEZ, A. MEDINA-FLORES, M. PÉREZ-LÓPEZ Y C. MALDONADO-ZEPEDA

Tabla II. Cuantificación del espectro de la figura 2

Table II. Quantitative spectrum of the figure 2

\begin{tabular}{|c|c|c|c|c|c|}
\hline Elemento & Peso ( \%) & Atómico (\%) & Incertidumbre (\%) & Corrección & Factor-k \\
\hline$N(K)$ & 5,666 & 19,434 & $0,443 /$ & 0,259 & 4,045 \\
\hline $\operatorname{Cr}(\mathrm{K})$ & 0,888 & 0,822 & 0,091 & 0,991 & 1,376 \\
\hline $\mathrm{Mn}(\mathrm{K})$ & 0,000 & 0,000 & 100,000 & 0,993 & 1,451 \\
\hline $\mathrm{Fe}(\mathrm{K})$ & 92,151 & 78,781 & 0,809 & 0,994 & 1,480 \\
\hline $\mathrm{Ni}(\mathrm{K})$ & 0,631 & 0,381 & 0,093 & 0,996 & 1,592 \\
\hline $\mathrm{Mo}(\mathrm{K})$ & 0,661 & 0,579 & 0,86 & 0,998 & 4,581 \\
\hline
\end{tabular}

\subsection{Caracterización del nitruro $\varepsilon-\mathrm{Fe}_{2-3} \mathrm{~N}$}

La figura 4 muestra una imagen de HRTEM de la fase $\varepsilon-\mathrm{Fe}_{2-3} \mathrm{~N}$ después de aplicarle la IFFT en forma de placa obtenida sobre la capa nitrurada; su correspondiente FFT se muestra en la misma figura, en la parte superior derecha, el cual es característico de una estructura hexagonal con sus principales planos marcados y sus correspondientes valores. Al indexar la FFT se determino que la imagen presenta un eje de zona cercano a la orientación $[1,2,0]$.

En la figura 4 sólo se observa la reflexión del plano (002), debido a que el microscopio no pude

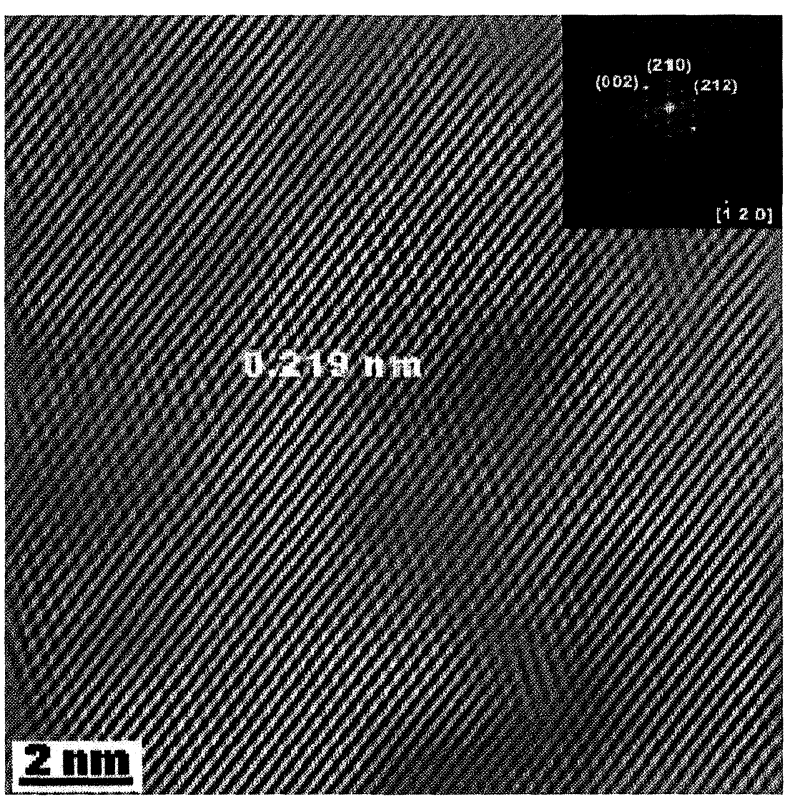

Figura 4. Imagen de la IFFT obtenida por HRTEM del nitruro $\varepsilon-\mathrm{Fe}_{2-3} \mathrm{~N}$ mostrando un distanciamiento interplanar de $0,219 \mathrm{~nm}$, que corresponde a la distancia entre el plano (002) y Transformada rápida de Fourier (FFT) en la orientación $[T, 2,0]$.

Figure 4. (a) IFFT HRTEM image of the $\varepsilon-\mathrm{Fe}_{2-3} \mathrm{~N}$ nitride showing an interatomic distance of $0,219 \mathrm{~nm}$ that correspond to the distance of the (002) plane and Fast Fourier Transform (FFT) in the $[T, 2,0]$ orientation. resolver distancias más pequeñas para las demás reflexiones y su respectivo patrón de FFT muestra claramente dos puntos principales producidos por la reflexión (002). En la figura obtenida por HRTEM se midieron las distancias interplanares, las cuáles presentan un valor de 0,219 nm, que coincide con el establecido en las tablas JCPDSICDD para este nitruro.

De igual manera, en la figura 5 que es el espectro de energía dispersiva (EDS) de la figura 4, se observan los picos del nitrógeno y hierro. La cuantificación del espectro muestra que el contenido de nitrógeno es de 25,364 \% atómico, lo cual nuevamente está de acuerdo a lo expuesto por R.C. Rhul y M Cohen.

\subsection{Imágenes mostrando coherencia entre los nitruros $\varepsilon-\mathrm{Fe}_{2-3} \mathrm{~N}$ y $\gamma^{\prime}-\mathrm{Fe}_{4} \mathrm{~N}$ con la matriz}

La imagen 6 (a) presenta una imagen obtenida mediante HRTEM sobre la zona de difusión, donde se observa la coexistencia de diferentes fases marcadas con diferentes números. La zona marcada con el número 1 en la imagen 6 (a) presenta el contorno de una partícula con un distanciamiento interplanar de 0,202 $\mathrm{nm}$, cuya distancia pertenece a una partícula de hierro en el plano (110); la zona

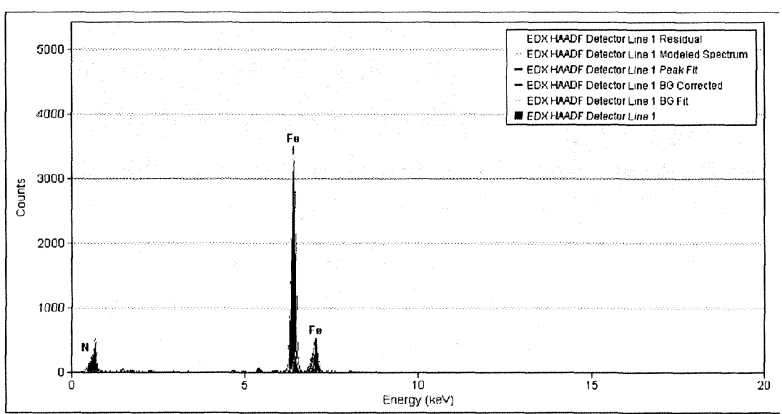

Figura 5. Espectro de nanoanálisis de la fase $\varepsilon-\mathrm{Fe}_{2-3} \mathrm{~N}$.

Figure 5. Nanoanalysis espectrum of the $\varepsilon-\mathrm{Fe}_{2-3} \mathrm{~N}$ phase. 

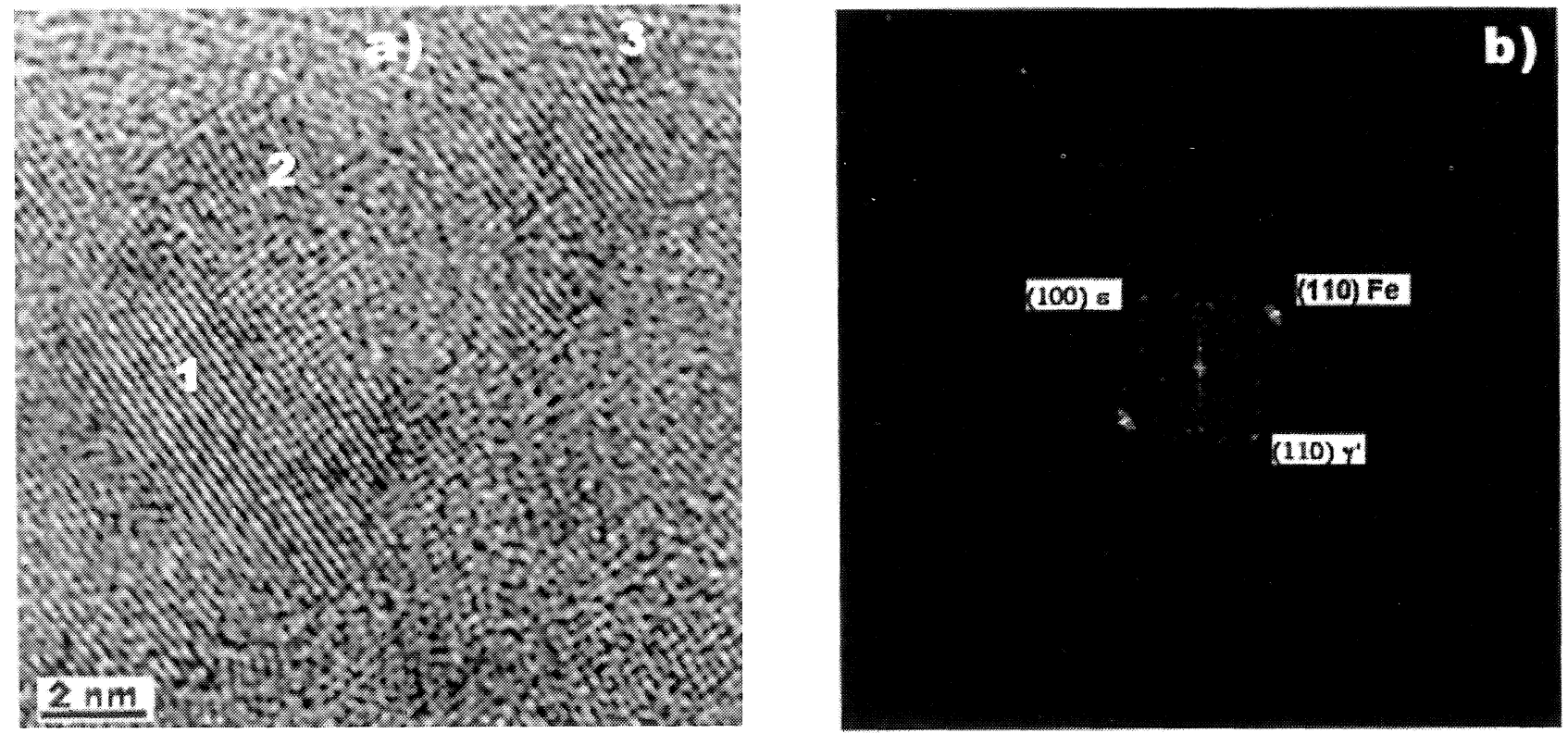

Figura 6. a) Imagen obtenida por HRTEM en la cual se observa la presencia y coherencia entre diferentes nitruros y matriz. b) Transformada rápida de Fourier (FFT) mostrando las diferentes frecuencias producidas por los diferentes cristales con distancias interplanares de 0,202 nm, 0,234 nm y 0,268 nm para los cristales de $\mathrm{Fe}-\alpha, \varepsilon-\mathrm{Fe}_{2-3} \mathrm{~N}$ y $\gamma^{\prime}-\mathrm{Fe}_{4} \mathrm{~N}$ respectivamente.

Figure 6. a) HRTEM image showing coherency between different nitrides and matrix. b) Fast Fourier Transform (FFT) showing the different frequencies produced for the different crystals whit interatomic distances of 0,202 $\mathrm{nm}, 0,234 \mathrm{~nm}$ y $0,268 \mathrm{~nm}$ for the crystal of $\mathrm{Fe}-\alpha, \varepsilon-\mathrm{Fe}_{2-3} \mathrm{~N}$ y $\gamma^{\prime}-\mathrm{Fe}_{4} \mathrm{~N}$ respectively.

Tabla II. Cuantificación del espectro de la figura 4

Table II. Quantitative spectrum of the figure 4

\begin{tabular}{|c|c|c|c|c|c|}
\hline Elemento & Peso ( \%) & Atómico (\%) & Incertidumbre (\%) & Corrección & Factor-k \\
\hline$N(K)$ & 7,72 & 25,364 & 0,021 & 0,259 & 4,045 \\
\hline $\mathrm{Cr}(\mathrm{K})$ & 0,847 & 0,983 & 0,072 & 0,991 & 1,376 \\
\hline $\mathrm{Mn}(\mathrm{K})$ & 0,000 & 0,000 & 100,000 & 0,993 & 1,451 \\
\hline $\mathrm{Fe}(\mathrm{K})$ & 90,684 & 73,189 & 0,832 & 0,994 & 1,480 \\
\hline $\mathrm{Ni}(\mathrm{K})$ & 0,662 & 0,372 & 0,030 & 0,996 & 1,592 \\
\hline $\mathrm{Mo}(\mathrm{K})$ & 0,0850 & 0,092 & 0,018 & 0,998 & 4,581 \\
\hline
\end{tabular}

marcada con el número 2 presenta el contorno de una partícula con un distanciamiento interplanar de 0,234 nm, que corresponde a un distanciamiento interplanar de la fase $\varepsilon-\mathrm{Fe}_{2-3} \mathrm{~N}$ en el plano (100); y la zona marcada con el número 3 tiene distancias interplanares con un valor de 0,268 nm que corresponde a un espaciamiento interplanar de la fase $\gamma^{\prime}-\mathrm{Fe}_{4} \mathrm{~N}$ en el plano (110). En esta imagen se observa coherencia entre los nitruros y con la matriz, pero no se observó límites de transformación.

La figura 6 (b) muestra el correspondiente FFT de la figura 6 (a), en el cual se pueden observar las diferentes frecuencias producidas por las diferentes fases.
Del análisis de esta imagen se obtiene la siguiente relación de coherencia entre planos:

$$
\varepsilon-\mathrm{Fe}_{2-3} \mathrm{~N}(100)|| \gamma^{\prime}-\mathrm{Fe}_{4} \mathrm{~N}(110)|| \mathrm{Fe}(110)
$$

Durante el enfriamiento lento, se espera que la región de $\varepsilon-\mathrm{Fe}_{2-3} \mathrm{~N}$ en la muestra se descomponga en una mezcla de equilibrio de $\varepsilon-\mathrm{Fe}_{2.3} \mathrm{~N}$ y $\gamma^{\prime}-\mathrm{Fe}_{4} \mathrm{~N}$. Las observaciones que se presentan en esta imagen indican que la transformación ocurre durante el enfriamiento y que la que transformación se lleva acabo por movimientos de planos compactos, dónde los átomos de hierro y nitrógeno sufren un reacomodo ${ }^{[7]}$. 
La forma y tamaño de los precipitados dependen fuertemente del equilibrio químico en las interfases y de la estructura de cada fase.

\section{CONCLUSIONES}

Los resultados obtenidos por HRTEM muestran diferentes morfologías de los nitruros $\varepsilon-\mathrm{Fe}_{2-3} \mathrm{~N}$ y $\gamma^{\prime}-\mathrm{Fe}_{4} \mathrm{~N}$; la digitalización de imágenes permitió y facilitó el análisis de los nitruros formados determinándose la estructura cristalina y la coherencia que existe entre ellos con la matriz.

De los resultados de Microscopia Electrónica de Transmisión y de Alta Resolución, se establece que las relaciones de coherencia entre precipitados y matriz es:

(110) $\mathrm{Fe}-\alpha||(1001) \varepsilon-\mathrm{Fe}_{2-3} \mathrm{~N} \|(110) \gamma^{\prime}-\mathrm{Fe}_{4} \mathrm{~N}$ y $\left[\begin{array}{lll}1 & -1 & 0\end{array}\right] \mathrm{Fe}-\alpha||\left[\begin{array}{llll}1 & 0 & -1 & 0\end{array}\right] \varepsilon-\mathrm{Fe}_{2-3} \mathrm{~N} \|\left[\begin{array}{lll}0 & 1 & 1\end{array}\right] \mathrm{Fe}_{4} \mathrm{~N}$

Los nanoanálisis muestra que el contenido de nitrógeno, está dentro de los valores permitidos para la fase $\gamma^{\prime}-\mathrm{Fe}_{4} \mathrm{~N}$ y $\varepsilon-\mathrm{Fe}_{2.3} \mathrm{~N}$, lo cual corrobora lo obtenido anteriormente.

\section{REFERENCIAS}

[1] B. Podgornik, J. Vizintin, O. Wanstrand, M. Larsson, S. Hogmark, H. Ronkainen y K. HolmberG. Wear 249 (2001) 254-259.

[2] U. Figueroa, J. Oseguera y P. Schabes, Proc. $2^{\text {nd }}$ Int. Conf. Carburizing and Nitriding with Atmospheres. ASM Cleveland, Ohio, USA (1995) 365-369.

[3] M.J. Yacamán Y J.A. Ascencio, Handbook of Nanostructured Máterials, Pergamon Press, 1999, pp. 135. 143.

[4] D.B. Williams y C.B. CARTER, Transmission Electron Microscopy: A Textbook for Materials Science, Plenum Press, New York, EE.UU., 1996.

[5] J.A. Ascencio, C. Gutiérrez, M. Espinoza, M. Marín, S. Tehuacanero, C. Zorrilla y M. J. Yacamán, Surf. Sci. 396 (1998) 349-368.

[6] W. KOHN and L.J. SHAM, Phys. Rev. 140 (1965) A1.133A1.138

[7] D. Gerardin, J.P. Mornirolli, H. Michel y M. Gantois, J. Mater. Sci. 16, (1981) 159. 